\title{
Interaction Between Bound Cupric Ion and Spin-Labeled Cysteine $\beta-93$ in Human and Horse Hemoglobins
}

\author{
William E. Antholine, Fumito Taketa \\ Departments of Radiology and Biochemistry, Medical College of Wisconsin, \\ Milwaukee
}

J. T. Wang

Department of Chemistry, Duquesne University, Pittsburgh, Pennsylvania

\section{P. T. Manoharan ${ }^{\mathbf{1}}$ and Joseph M. Rifkind}

Laboratory of Cellular and Molecular Biology, National Institute on Aging, National Institutes of Health, Gerontology Research Center, Baltimore, Maryland

\begin{abstract}
The location of the various copper binding sites for horse and human hemoglobin was probed using spin labels attached to the $\beta-93$ cysteine residue. Dipole-dipole interactions between the spin label and bound copper produce a decrease in the amplitude of the spin label spectrum which was used to estimate the $\mathrm{Cu}$ (II) spin label distance. By comparing the results with horse and human hemoglobin at 298 and $77 \mathrm{~K}$ four different $\mathrm{Cu}$ (II) binding sites were identified. The low affinity horse hemoglobin site with the sulfhydryl blocked (site 1) was found to be located 10-13 $\AA$ from the sulfhydryl spin label on the surface of the molecule. Only with a free sulfhydryl is the site (site 2) in the pocket between the $\mathrm{F}$ and $\mathrm{H}$ helices closer to the SH-group and the iron populated. It is site 2 which is responsible for the oxidation. In frozen solutions a Cu-nitroxide distance of about $17 \AA$ was determined with human hemoglobin. This distance is consistent with the previously postulated location of the "high affinity" human hemoglobin site near the amino terminus of the $\beta$-chain. At $298 \mathrm{~K}$ a much shorter Cu-nitroxide distance of about $7 \AA$ was calculated for human hemoglobin. This shorter distance at higher temperature also correlated with a slightly smaller value of $g_{11}$ and $A_{11}$ for the Cu(II) ESR spectrum. It is postulated that in solution cross-linking between nitrogenous ligands in the region of the amino terminus of one $\beta$ -
\end{abstract}

Address reprint requests to Dr. Joseph M. Rifkind, Laboratory of Cellular and Molecular Biology, NIH/NIA, Gerontology Research Center, Baltimore, Maryland 21224.

1 On leave from the Indian Institute of Technology, Madras, India. 
chain and the carboxyl tcrminus of the other $\beta$-chain can explain this shorter distance. This cross link could involve histidine $\beta-143$, which is one of the ligands thought to be also involved in site 1. Binding to the "high-affinity" site in solution thus stabilizes the "low-affinity" site 2 relative to site 1 explaining the reported interaction between the "high-affinity" and "low-affinity" sites.

\section{INTRODUCTION}

Studies on the binding of cupric ion to human hemoglobin have shown that a "highaffinity" site as well as several "low-affinity" sites can be identified using ESR techniques [1-5]. Not all hemoglobins contain the "high-affinity" site. Hemoglobins like those of horse and cat lack such a site, apparently because they do not contain an important histidine residue at position $2 \beta$, adjacent to the amino terminal residue of the $\beta$ subunit. The "high-affinity" site is evidently located in this region of the protein, and it appears to involve the donor nitrogen of the $\alpha$-amino group of the amino terminal residue, as well as the imidazole group of $\beta$-2 histidine. ESR studies indicate a total of four nitrogens producing a square planar configuration of atoms for the tight binding of copper [6]. Two moles of cupric ions are bound per mole of hemoglobins that contain these sites, without oxidation of the hemes. Selective oxidation of their $\beta$ hemes ensues only when more than two moles of cupric ion are added. In contrast, rapid oxidation of $\beta$ hemes occurs when less than two moles of copper are added to hemoglobins that lack the "high affinity" cupric ion binding site. The oxidation of the $\beta$ hemes in both classes of hemoglobins is associated with binding of cupric ion at one or more of the "low-affinity" sites.

The main purpose of this paper is to probe the location of these copper binding sites by spin labeling techniques. The amino acid residue cysteine $93 \beta$ is adjacent to the proximal histidine residue $92 \beta$ within the $\beta$-subunit. This sulfhydryl group is readily modified with nitroxide spin labels to provide the basis to probe the copper binding sites. The addition of copper(II) ions to the labeled hemoglobins produces perceptible to dramatic decreases in the amplitude of the spectra due to the spin label with almost no change in the shape of the lines. This apparent decrease in the amplitude of the signal due to the spin label as a function of the hemoglobin/ $\mathrm{Cu}$ (II)-mole ratio is due to a strong dipole-dipole interaction [7]. According to the theory of Leigh [7], only those spins lying within a small range of orientations close to the "magic angle" produce amplitude variations without changing the width. Such a decrease in amplitude is hence inversely related to the distances between the concerned dipoles and can be used in conjunction with available crystallographic data to derive information about the location of the copper binding sites.

In the present work we find that in horse hemoglobin, without a "high-affinity" site, the distance between the bound $\mathrm{Cu}$ (II) and the nitroxide attached to cysteine $\beta-93$ falls within the range of $10-13 \AA$. This relatively short distance is consistent with the expected location of this site on the proximal side of the heme [3]. For human hemoglobin it is found that the distance to $\mathrm{Cu}$ (II) in frozen solution at $77 \mathrm{~K}$ using the same label is about $17 \AA$, consistent with the expected distance to the $\mathrm{N}$-terminal end of the $\beta$-subunit. However, at $298 \mathrm{~K}$ the distance between the $\mathrm{Cu}(\mathrm{II})$ and the nitroxide group is apparently reduced to $7 \AA$. This unexpected difference could be attributed to an alteration in the coordination of the "high-affinity" site at elevated temperatures.

Furthermore, the analysis of $\mathrm{Cu}(\mathrm{II})$ ESR spectra of various hemoglohins suggests a correlation between the presence of the "high-affinity" copper binding site and the strength of interaction between the heme iron and $\mathrm{Cu}$ (II) at the "low-affinity" copper 
binding site [2]. This suggests the possibility that the cupric ion bound at the "highaffinity" site indirectly affects the binding of additional cupric ions at the locus near the heme.

\section{MATERIALS AND METHODS}

Preparation of Spin-Labeled Hemoglobin. Fresh human blood was obtained from the Blood Bank and horse hemoglobin was defibrinated pooled blood from Bioquest. Hemolysates werc prepared and hemoglobin concentration detcrmined by conventional procedures. Hemoglobin solutions were stripped free of phosphates [1], concentrated to about $10 \%$ by ultrafiltration (Amicon, PM-10 membrane), and used within one week after drawing the blood (human). Hemoglobin was oxidized to form methemoglobin by a fivefold excess of ferricyanide, which was then removed by exhaustive dialysis. Fivefold excess of spin labels per mole of hemoglobin $(2 \mathrm{~mm})$ were reacted for $1.5 \mathrm{hr}$ at room temperature and then dialyzed exhaustively versus 0.1 $M$ phosphate buffer, pH 7.0 in a cold room at $4^{\circ} \mathrm{C}$. Spin labels were obtained from Syva and/or Aldrich Chemicals. The spin labels used in our experiments are as follows:
I, 4-(2-maleimido)-TEMPO
II, 4-(2-iodoacetamido)-TEMPO
III, 3-maleimido-PROXYL
IV, 3-(maleimidomethyl)-PROXYL
V, 3-[2-(2-maleimido ethyl)carbamoyl]-PROXYL
VI, 3-[(3-maleimido propyl)carbamoyl]-PROXYL

While labels I and II were studied with human and horse hemoglobins, labels III-VI were only used with horse hemoglobin in order to understand the effect of increasing the chain length of the label on copper interactions.

Preparation of NEM-Hemoglobins. The hemoglobin was incubated with a small excess of NEM. The unreacted NEM was removed by gel-filtration. 4,4 Dithiopyridine was then used to check for unreacted sulfhydryl groups. The reactions with NEM were found to have blocked $>98 \%$ of the free SH groups.

ESR analysis was conducted with a Varian E-109 Century Series $X$-band spectrometer located at the National Biomedical ESR Center and a JEOL-JES-ME $X$ band spectrometer at NIH/NIA. Spectra from frozen samples were obtaincd by supporting a frozen icicle sample $(3 \mathrm{~cm}$ long and $4 \mathrm{~mm}$ diam) in the cavity with a finger Dewar filled with liquid nitrogen. Spectra at room temperature were obtained from samples in a standard flat cell.

\section{RESULTS}

\section{Effect of Cu on Spin Label Signal Intensities}

The initial studies on identifying the copper binding site in horse hemoglobin were done by monitoring the reduction in the EPR intensity of spin-labeled horse methemoglobin by systematic addition of exogenous copper(II) ion. Here, $\mathrm{Cu}$ (II) 
binding at the "low-affinity" site in the proximity of the heme and cysteine $\beta-93$ sulfhydryl group to which the spin label is attached is responsible for the decrease in amplitude of the spin label signal. Figure 1 shows the decrease in amplitude of various spin labels (III-VI) of differing length attached to the $\beta-93$ sulfhydryl group of horse methemoglobin as a function of added $\mathrm{Cu}(\mathrm{II})$ ion. This reduction in the signal amplitude of the spin label can be used to calculate the distance between the metal ion and nitroxide radical according to the theory of Leigh [6] if the spin lattice relaxation time, $T_{1}$, for cupric ion is known. The relation of Leigh [7]

$$
C=g \beta M^{2} T_{1} / r^{6} h
$$

was used to calculate the distance $r$ in these spin-labeled hemoglobins. It was found that the use of a relaxation time of $T_{1}=10^{-9} \mathrm{sec}$ [8] gives values of 9.4-13.5 $\AA$ for $r$ with the shorter distance associated with the longer labels.

It is further noted [3] that the longest label close to the $\mathrm{Cu}$ (II) site has an EPR spectrum representative of a weakly immobilized component and is presumably located on the surface of the $\beta$-subunit. On the other hand the shortest labels somewhat

FIGURE 1. ESR amplitude profile of the spin-labeled horse hemoglobins as a function of added copper for various labels. $O$, 3-(maleimido)PROXYL; $\square$, 3-(maleimidomethyl)PROXYL; 0, 3-[2-(2-maleimidoethyl)carbamoyl]PROXYL; $A, 3-[(3-$ maleimidopropyl)carbamoyl]PROXYL.

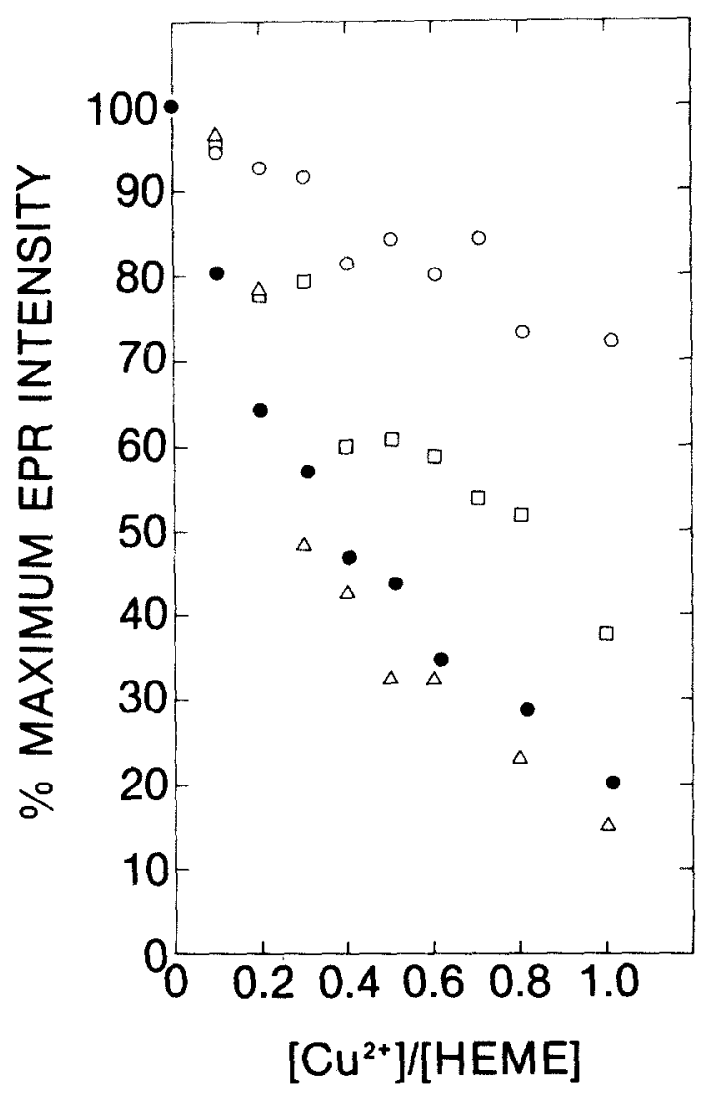


farther from the $\mathrm{Cu}$ (II) site revealed a spectrum corresponding to a strongly immobilized component located in the pocket between the $\mathrm{F}$ and $\mathrm{H}$ helices.

Figures $2 \mathrm{a}$ and $2 \mathrm{~b}$ show a comparison of the reduction of the spin label amplitude as a function of added $\mathrm{Cu}^{2+}$ to labeled horse and human methemoglobins with 4-(2maleimido) TEMPO and 4-(2-iodoacetamido)-TEMPO, respectively. While the initial slope in these human hemoglobin experiments is dependent on the distance between the "high-affinity" copper(II) and spin label, the slope of the horse hemoglobin experiment is suggestive of the distance between the "low-affinity" site involved in oxidation and the spin label. Surprisingly the figure shows that for both labels the $\mathrm{Cu}$ (II) distance is shorter in human hemoglobin than horse hemoglobin, suggesting that the "high-affinity" site is actually closer to the sulfhydryl than the "low-affinity" site. The $\mathrm{Cu}(\mathrm{II})$-nitroxide distances derived from the use of Leigh's equation (1) using a room-temperature spin-lattice relaxation time $T_{1}=10^{-9} \mathrm{sec}$ [8] for copper indicate an approximately $3 \AA$ shorter distance for human hemoglobin than horse hemoglobin.

Figures $3 \mathrm{a}$ and $3 \mathrm{~b}$ show a comparison of the variation of ESR signal amplitudes of human and horse methemoglobins labeled with 4-(2-maleimido)-TEMPO at two different temperatures.

For horse hemoglobin, the effect of freezing and lowering the temperature to $77 \mathrm{~K}$ is seen to be minimal for the initial binding site. In order to compare the distances at both temperatures it is necessary to take into account the expected increase in $T_{1}$ at lower temperatures. Assuming an order of magnitude increase in $T_{1}$ at $77 \mathrm{~K}$ to $10^{-8}$, the calculated $\mathrm{Cu}(\mathrm{II})$ nitroxide distance increases from $10 \AA$ at $298 \mathrm{~K}$ to $13 \AA$ at $77 \mathrm{~K}$. This small difference could result from the uncertainty in the magnitude of the increase in $T_{1}$ at $77 \mathrm{~K}$ or from a decrease in conformational mobility of both the protein and the label which alters the metal-nitroxide distance. However, for human hemoglobin a

FIGURE 2. (a) ESR amplitude change in human hemoglobin ( $\square$ ) and horse hemoglobin (a) spin labeled with 4-(2-maleimido)TEMPO at a temperature of $300 \mathrm{~K}$. (b) ESR amplitude change in human ( $\square$ ) and horse $(\mathbb{a})$ hemoglobins spin labeled with 4-(2-iodoacetamido)TEMPO.

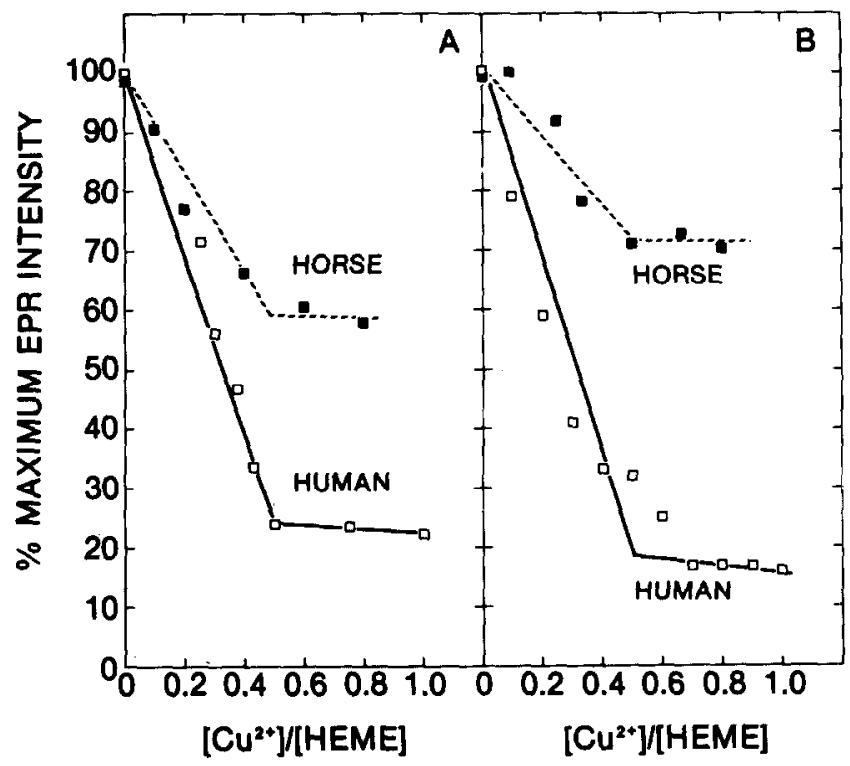




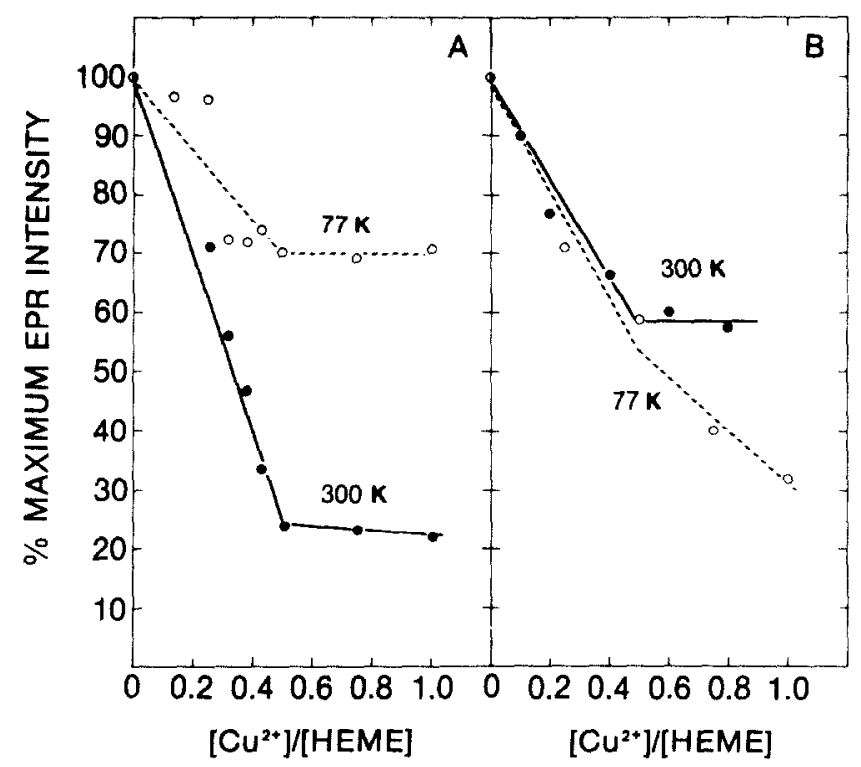

FIGURE 3. (a) Variation of ESR spectral intensity of human hemoglobins labeled with 4-(2maleimido)TEMPO as a function of added $\mathrm{Cu}^{2+}$ ion. $\bigcirc, 77 \mathrm{~K}$ and $\odot, 300 \mathrm{~K}$. (b) Variation of ESR spectral intensity of horse hemoglobin labeled with 4-(2-maleimido)TEMPO as a function of added $\mathrm{Cu}^{2+}$ ion. $\bigcirc, 77$ $\mathrm{K}$ and, $300 \mathrm{~K}$.

much larger difference is observed between 298 and $77 \mathrm{~K}$ (Fig. 3) and the calculated increase in the metal-nitroxide distance from $7 \AA$ (strong interaction) at $298 \mathrm{~K}$ to about $17 \AA$ (weak interaction) at $77 \mathrm{~K}$ cannot be attributed to either conformational mobility or any uncertainty in the $T_{1}$. It was further noted that the spin Hamiltonian parameters of bound $\mathrm{Cu}^{2+}$ in human hemoglobins does change, though to a small extent, when cooled from 298 down to $77 \mathrm{~K}$ (Table 1 and Fig. 4).

Interestingly, the weak interaction observed at $77 \mathrm{~K}$ is consistent with the expected distance between the nitroxide and the $\mathrm{N}$-terminal region of the $\beta$-chain, where the high-affinity human site is thought to be located.

The saturation properties of the EPR signals due to the spin label 4-(2-maleimido)TEMPO in human hemoglobin were studied (Fig. 5) at these two temperatures, viz., 298 and $77 \mathrm{~K}$, both in the presence and absence of two equivalents of $\mathrm{Cu}(\mathrm{II})$. This experiment was necessary in order to prove that the fivefold reduction in the ESR intensity on the addition of copper to the labeled human hemoglobin, especially at room temperature, is not associated with an effect of $\mathrm{Cu}$ (II) on the relaxation of the

TABLE 1. ESR Parameters for $\mathrm{CuHb}$

\begin{tabular}{lccc}
\hline & $\begin{array}{c}\text { Temperature } \\
(\mathrm{K})\end{array}$ & $g_{11}$ & $\begin{array}{c}A_{11} \\
\left(\mathrm{~cm}^{-1}\right)\end{array}$ \\
\hline $1.8 \mathrm{eq} . \mathrm{Cu}^{2+} / \mathrm{Hb}$ tetramer & 300 & 2.201 & $197 \times 10^{-4}$ \\
$1.8 \mathrm{eq} . \mathrm{Cu}^{2+} / \mathrm{Hb}$ tetramer & 77 & 2.207 & $207 \times 10^{-4}$ \\
\hline
\end{tabular}




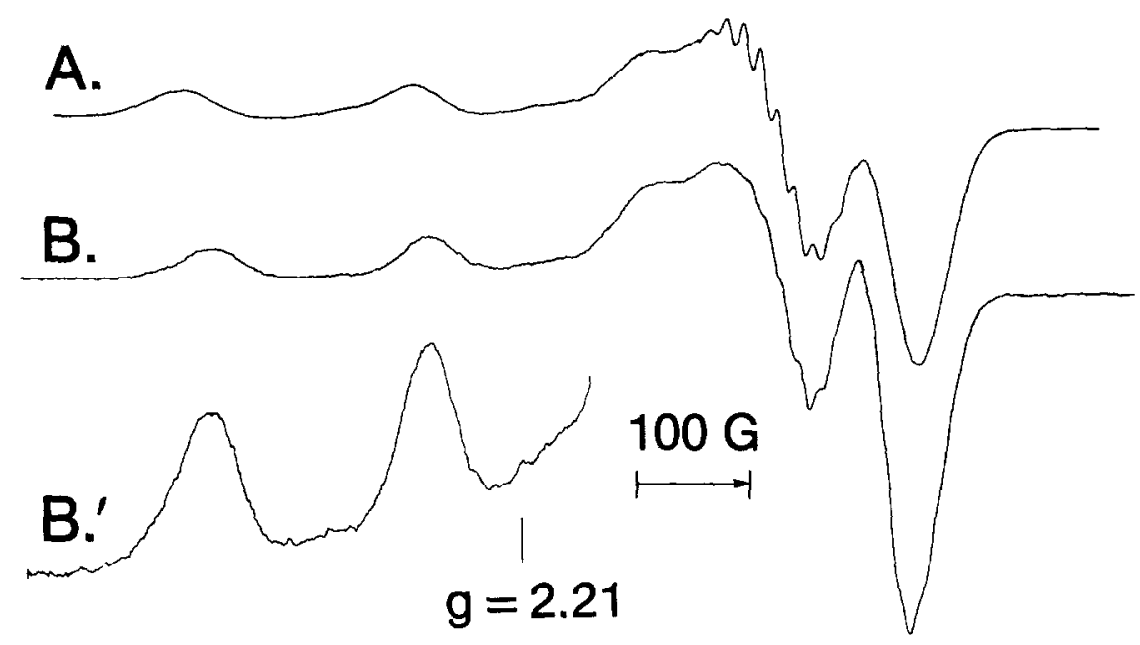

FIGURE 4. ESR spectra for $0.5 \mathrm{mM}$ human hemoglobin plus two equivalents of cupric ion at (A) $77 \mathrm{~K}$ and $(B)$ room temperature. Spectrometer conditions for (A): $5 \mathrm{~mW}$ incident power, $5 \mathrm{G}$ mod. amp., 100 $\mathrm{kHz}$ mod. freq., time constant $0.3 \mathrm{sec}$, gain $2 \times 10^{5}$. Spectrometer conditions for (B): incident power 200 $\mathrm{mW}, 5 \mathrm{G}$ mod. amp., $100 \mathrm{kHz}$ mod. freq., time const. $0.3 \mathrm{sec}$, gain $8 \times 10^{2}$ and for (B) the gain is $4 \times$ $10^{3}$.

spin label and its saturation properties. It can be seen from this figure that cupric ion has little effect on the relaxation of the spin labels. At room temperature no saturation of the spin label signals was detected, and for frozen samples the spin label signal begins to saturate only below $28 \mathrm{~dB}$ both in the presence and absence of $\mathrm{Cu}$ (II).

\section{Effect of Cu on ESR Spectrum of Nitroxides Bound to Hemoglobin}

Figure 6 shows the effect of binding copper to the initial binding site on the ESR spectrum of the 4-(2-maleimido)-TEMPO bound to horse and human hemoglobin. For horse hemoglobin essentially no change in the spectrum is observed, even though the intensity decreases. On the other hand, for human hemoglobin there is the appearance of a partially immobilized spin label signal at room temperature in addition to the strongly immobilized signal observed in the absence of copper.

\section{Effect of Blocking the $\beta-93$ Sulfhydryl on the Interaction of $\mathrm{Cu}(\mathrm{II})$ with Hemoglobin}

For human hemoglobin, no effect other than a slight broadening was seen when the spectrum of $\mathrm{Cu}$ (II) bound at the $\mathrm{Cu} /$ heme ratio of 0.5 was compared after or before having reacted the $\beta-93$ sulfhydryl with spin labels. For horse hemoglobin, the binding site in the absence of spin labels consists of an equilibrium between a site on the surface where $\mathrm{Cu}$ initially binds and a second site resulting from a conformational change from which the actual oxidation takes place [9]. As previously shown for NEM [9], after reacting with spin label only the initial site is detected. This difference between human and horse hemoglobin is also seen in equilibrium binding studies of copper (Fig. 7).

Human hemoglobin has a copper affinity which is approximately an order of 


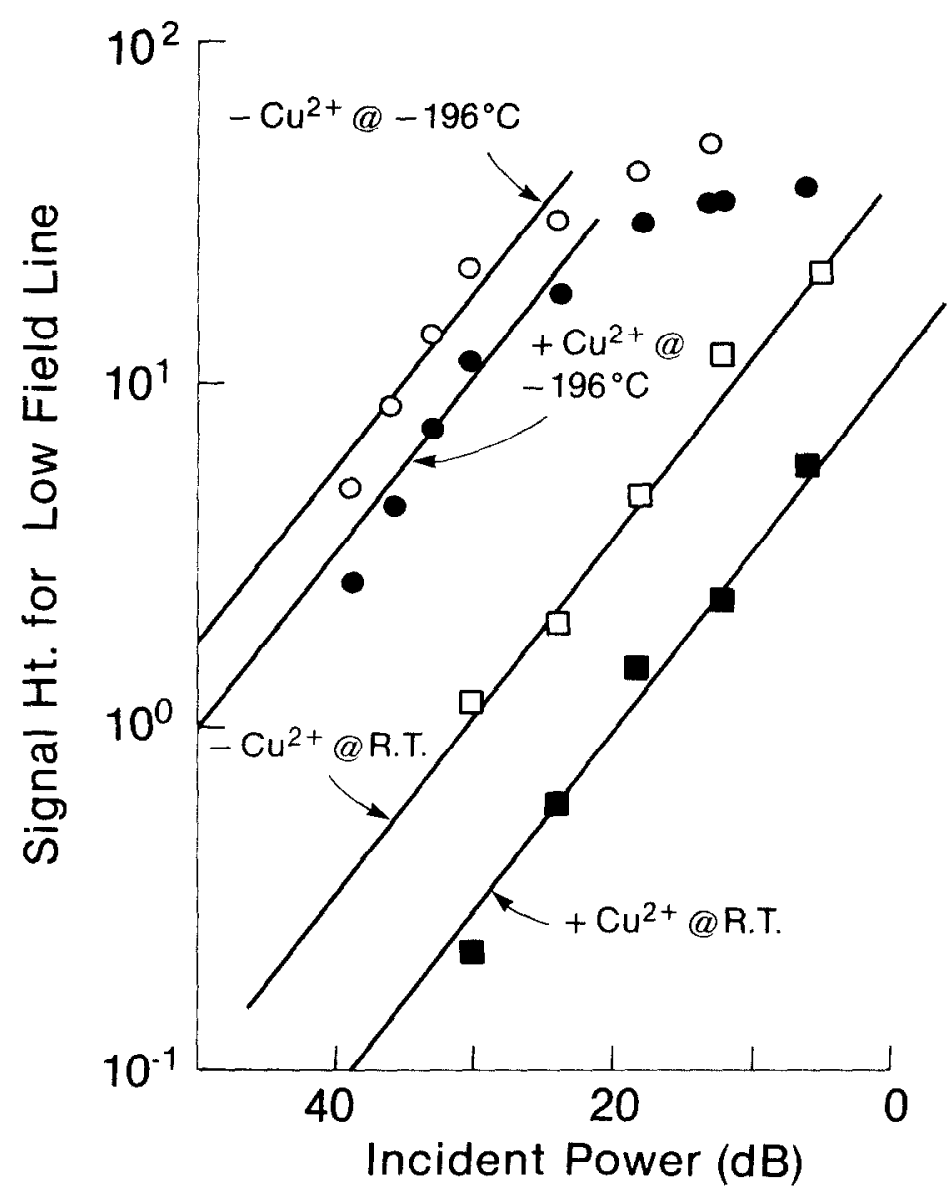

FIGURE 5. Signal height for low-field ESR line versus microwave power (dB) for spin-labeled hemoglobin at rom temperature in the presence $(\square)$ and absence $(\square)$ of two equivalents of cupric ion, and at $77 \mathrm{~K}$ in the presence of $(0)$ and absence of two eqivalents of cupric ion (O).

magnitude higher than that of horse hemoglobin. Consistent with the $\mathrm{Cu}$ (II) ESR results, the effect of reaction with NEM on the binding of copper to horse and human hemoglobin reveals that the reaction with NEM produces a small but significant decrease in the affinity of horse hemoglobin for copper, while the binding of copper to human hemoglobin is unaffected at low concentrations when binding takes place at the "high-affinity" site. Interestingly, Figure 7 also shows that at higher concentrations of copper, the reaction with NEM has a very appreciable effect on the binding of $\mathrm{Cu}(\mathrm{II})$ to human hemoglobin. Thus, NEM has a much greater effect on binding of $\mathrm{Cu}$ (II) to the "low-affinity" site of human hemoglobin than the $20 \%$ decrease found for horse hemoglobin.

\section{SCUSSION}

Most of the earlier observations on the binding of copper to hemoglobins [1-6] reveal that horse and cat hemoglobins have one general area of binding which is also 

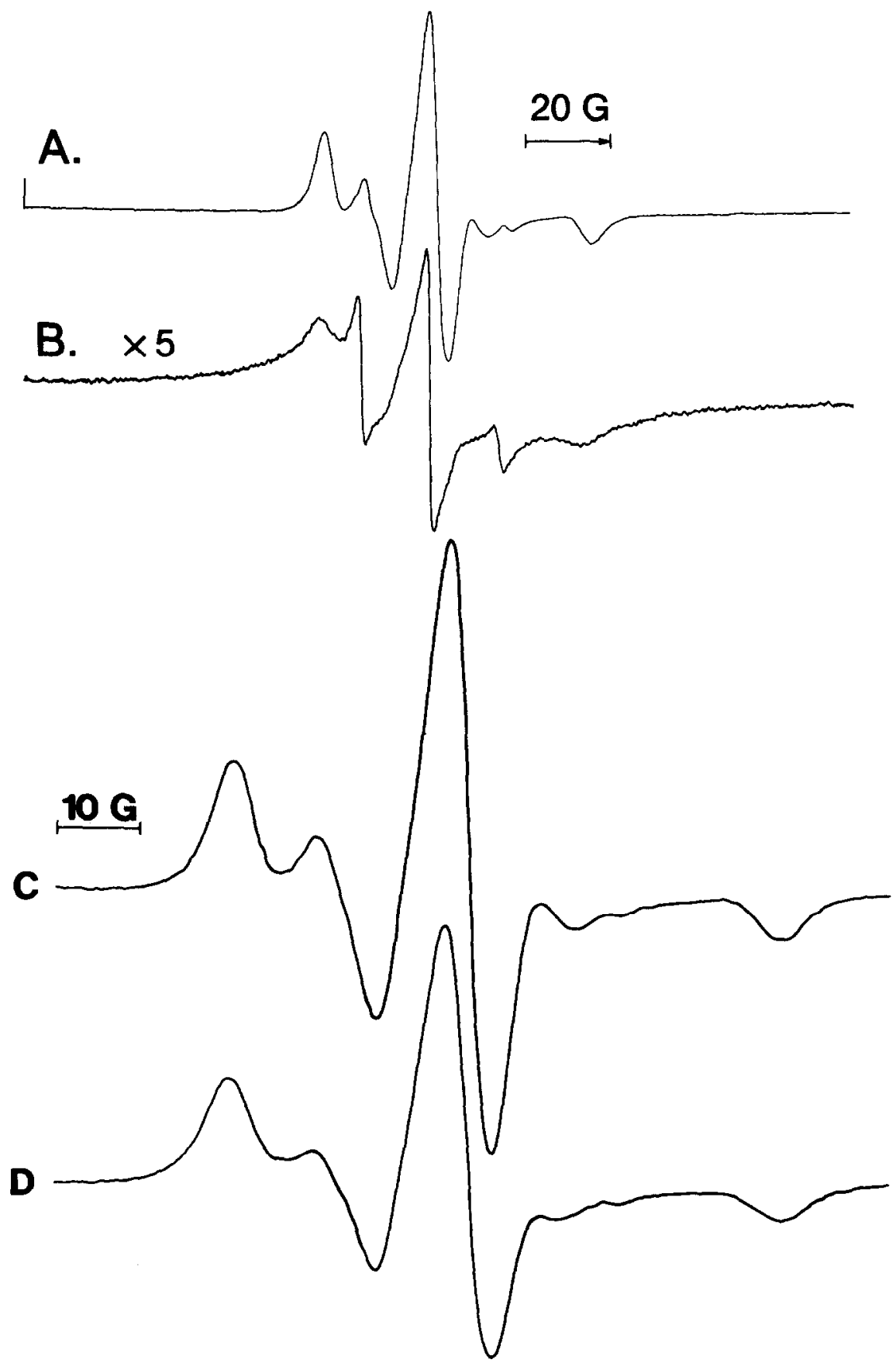

FIGURE 6. Room temperature ESR spectra for spin-labeled human and horse hemoglobin in the absence and presence of two equivalents of cupric ion. (Identical spectra were obtained when hemoglobin concentration was varied from $0.5 \mu \mathrm{M}$ to $0.7 \mathrm{mM}$ ). Spectrometer conditions: $2.0 \mathrm{G}$ mod. amp., $100 \mathrm{kHz}$ mod. freq., time const. $0.3 \mathrm{sec}$, (A) human hemoglobin in the absence of copper; (B) human hemoglobin in the presence of copper, gain $5 \times$ that of $A$; (C) horse hemoglobin in the absence of copper; (D) horse hemoglobin in the presence of copper, same gain as $C$. 


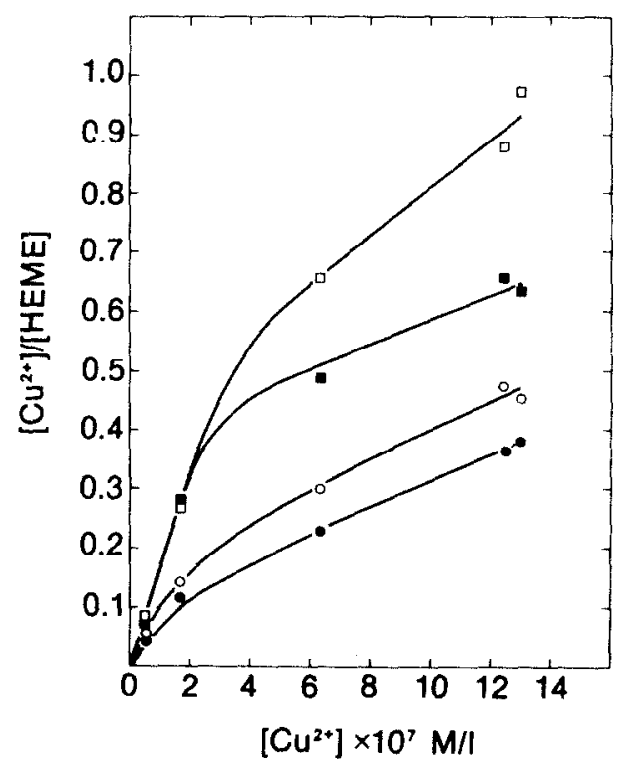

FIGURE 7. Binding of copper to human and horse hemoglobins before and after treatment with NEM $\square$, Human hemoglobin; $\mathbf{D}$, NEM + human hemoglobin; $\bigcirc$, horse hemoglobin; $\odot$, NEM + horse hemoglobin.

associated with the oxidation of the $\beta$-chains by copper. On the other hand, the human hemoglobin has an additional site involving one copper for every two hemes. The latter site has a higher copper affinity than the one in horse hemoglobin but is not associated with oxidation. This site, present only in hemoglobins with $\beta-2$ histidine, is known as the "high-affinity" or tight-binding site, while the other site, present in all known mammalian hemoglobins, is known as the "low-affinity" site.

\section{The Low-Affinity Site}

Previous studies indicate that this site is in the region of the $\beta-93$ sulfhydryl and that histidine $\beta-143$ as well as a nearby glutamic acid or aspartic acid residue may be involved in the coordination [3]. The short distance, $9-13.5 \AA$, from the nitroxide labels to the $\mathrm{Cu}(\mathrm{II})$ binding site confirms that this site is in the region of the $\beta-93$ cysteine residue. The comparison of the different spin labels indicates shorter $\mathrm{Cu}$ distances for the longer labels which we expected to lie on the surface of the protein out of the pocket between the $\mathrm{F}$ and $\mathrm{H}$ helices. This suggests that the $\mathrm{Cu}(\mathrm{II})$ binding site is not in the pocket but actually on the surface of the molecule, perhaps in the region of the $\beta-143$ histidine residue.

The failure to observe an effect on the shape of the maleimide spin label spectrum as a result of $\mathrm{Cu}$ (II) binding to horse hemoglobin (Fig. 6) is consistent with the conclusion that the $\mathrm{Cu}(\mathrm{II})$ is bound to the surface where it can produce a dipolar effect on the label but does not directly affect the mobility of the nitroxide label located in the pocket.

It should, however, be recognized that the "low-affinity" site, probed by the spin labels, corresponds to the initial rapidly bound $\mathrm{Cu}$ site with $g_{11}=2.28$ and $A_{11}=173$ 
$\times 10^{-4} \mathrm{~cm}^{-1}$ (site 1). The site actually responsible for oxidation with $g_{11}=2.21$ and $A_{11}=180 \times 10^{-4} \mathrm{~cm}^{-1}$ (site 2) requires a subsequent conformational change and is not found when the sulfhydryl group is blocked [9]. The $20 \%$ decrease in affinity of $\mathrm{Cu}(\mathrm{II})$ for horse hemoglobin when the $\beta-93$ sulfhydryl group is blocked by NEM (Fig. 7) is indicative of a greater $\mathrm{Cu}(\mathrm{II})$ affinity for site 2 than site 1.

Recent studies indicate strong metal-metal interactions between the low-affinity site of $\mathrm{Cu}$ (II) and the heme iron [2] as well as $\mathrm{Cu}$ (II)-Cu(II) interactions when $\mathrm{Cu}$ (II) is bound to the low-affinity site in $\mathrm{Cu}$ (II) substituted hemoglobin [5]. These results require that the $\mathrm{Cu}$ (II) be $<10 \AA$ from the heme iron. These studies on human hemoglobin with unreacted $\beta-93$ cysteine presumably probe site 2 (see below), which may be in the pocket between the $\mathrm{F}$ and $\mathrm{H}$ helices closer to the SH-group and closer to the iron.

\section{The High-Affinity Site}

It has been proposed earlier [3] that the presence or absence of a histidine residue at $\beta$ 2 determines whether a hemoglobin possessed or lacked a pair of tight binding sites for cupric ion. ESR data are consistent with the sites being comprised of four nitrogens in a configuration which is either approximately square planar or possibly pyramidal square planar [6]. A similar cupric ion binding site on human serum albumin involves the $\alpha$-amino nitrogen of the $\mathrm{N}$-terminal aspartic acid residue, two intervening peptide nitrogen atoms, and the imidazole nitrogen of the histidine residue in the third position [10].

In addition to the $\beta$-2 imidazole, the $\alpha$-amino group of the $\mathrm{N}$-terminal valine residue is implicated by recent studies investigating the oxidation of human hemoglobin where the $\alpha$-amino group is blocked [11]. Other nitrogen ligands in this region could involve peptide nitrogens or other nearby amino acids such as asparagine $\beta-80$, lysine $\beta-82$, or lysine $\beta-132$ [12].

The dipolar broadening experiments at $77 \mathrm{~K}$ for 4-(2-maleimido)-TEMPO labeled human hemoglobin (Fig. 3a) indicate a distance from the nitroxide group to the first bound $\mathrm{Cu}^{2+}$ in the "high-affinity" site of about $17 \AA$. This distance is consistent with the distance between the above postulated location (site 3) of the "high-affinity" $\mathrm{Cu}^{2+}$ binding site and the nitroxide group reacted with the $\beta-93$ sulfhydryl group. However, the room temperature dipolar broadening experiments for human hemoglobin with both 4-(2-maleimido)-TEMPO and 4-(2-iodoacetamido)-TEMPO (Figs. 2 and 3) indicate much shorter distances of 6-7 $\AA$, which are not consistent with this binding site.

The possibility that the $\mathrm{Cu}$-nitroxide interaction in the frozen sample at $77 \mathrm{~K}$ might not be the same as for a hemoglobin solution at $298 \mathrm{~K}$ was considered. The $T_{1}$ 's for copper have not been independently determined. Thus, the assumptions concerning the values of the $T_{1}$ 's must be approximately correct or calculations using Leigh theory are only qualitative. The effect of the greater mobility at $298 \mathrm{~K}$ on calculations based on the Leigh theory also need to be considered. Recent results indicate that the effective rigid limit ESR parameters obtained from liquid phase data may not be the same as the parameters obtained from the frozen state for low molecular weight complexes [13, 14]. The room temperature spectrum is sensitive to an averaging process that accounts for broadening and poorly resolved lines. However, the changes between 77 and 298 $\mathrm{K}$ found for horse hemoglobin using the same labels are small, suggesting that the Leigh theory may be valid even at room temperature. 
Recent studies by Tabak et al. [15] on the maleimido-PROXYL spin labels also indicate much shorter $\mathrm{Cu}$ (II)-nitroxide distances than expected for a $\mathrm{Cu}$ (II) bound to the $N$-terminal end of the $\beta$-chain. In order to explain their results they have postulated that the high-affinity $\mathrm{Cu}(\mathrm{II})$ binding site may involve the $\mathrm{N}$-terminal valine and $\beta-2$ histidine of one $\beta$-chain and the $\beta-143$ and $\beta-146$ histidines of the other $\beta$-subunit (site 4). This would place the $\mathrm{Cu}$ (II) binding site in the range of 8-13 $\AA$ from the sulfhydryl group in the second $\beta$-subunit and even closer to the nitroxide group. $\mathrm{Cu}$ (II) bound to such a site would produce large dipole broadening, which could be even greater than that found for the low-affinity site in horse hemoglobin. $\mathrm{Cu}$ (II) bound exclusively to the $N$-terminal region of the $\beta$-subunit would not be expected to alter the mobility of the spin label reacted with the $\beta-93 \mathrm{SH}$-group. However, the effect found on the spectrum (Fig. 6) for human hemoglobin can more readily be explained by crosslinking (site 4), which brings the $\mathrm{Cu}$ (II) closer to the pocket between the $\mathrm{F}$ and $\mathrm{H}$ helices and may perturb this pocket to an even greater extent than the $\mathrm{Cu}$ (II) bound to the "low-affinity" site 1 on the surface of the $\beta$-subunit.

The change in the dipolar effect as a function of temperature found for the highestaffinity $\mathrm{Cu}$ (II) binding site on human hemoglobin (Fig. 3), which is not found for horse hemoglobin, can be explained by a disruption of the cross-linking (site 4) at lower temperature in the frozen solution. Breaking the cross-link would result in the copper being localized on the $\mathrm{N}$-terminal end of the $\beta$-subunit further from the $\mathrm{SH}$ group. The low-temperature ESR of $\mathrm{Cu}$ (II) bound to this site (site 3) still indicates binding to four nitrogens. The $\beta-143$ and $\beta-146$ histidine residues presumably coordinated at room temperature are therefore presumably replaced by other nitrogenous ligands (see above). This change in the $\mathrm{Cu}$ (II)-hemoglobin complex could explain the small change in $g_{11}$ and $A_{11}$ of the $\mathrm{Cu}(\mathrm{II})$ bound to the high-affinity site at 77 and $298 \mathrm{~K}$ (Fig. 4, Table 1). A contribution from motion of the $\mathrm{Cu}$ (II) hemoglobin complex [16] may, however, also contribute to the small change in the Cu(II) ESR spectrum produced by freezing.

\section{Perturbation of Low-Affinity Site by Cu(II) Binding to High-Affinity Site}

In the early literature on the interaction of $\mathrm{Cu}(\mathrm{II})$ with hemoglobin, it was assumed that the "low-affinity" and "high-affinity" $\mathrm{Cu}$ (II) binding sites were independent of each other. With respect to oxidation, the presence of the high-affinity site which did not produced oxidation merely served to complex low levels of $\mathrm{Cu}$ (II) protecting the heme from oxidation [3, 4].

It has, however, been recently reported that the dipolar or exchange interaction between $\mathrm{Cu}$ (II) bound to the low-affinity site and the heme iron in methemoglobin is considerably greater for hemoglobins which possess the high-affinity site [2]. Such a perturbation is also suggested by the results of Figure 6, which indicate that binding $\mathrm{Cu}$ (II) to the "high-affinity" site (site 4) influences the spin label spectrum. This perturbation in the region of the spin label is not observed when copper is bound to horse hemoglobin where no "high-affinity" site is present. There is also evidence that binding $\mathrm{Cu}$ (II) to the "high-affinity" site enhances the affinity for the site (site 2) involved in the oxidation relative to that of site 1 (see above). This effect was seen in the appreciable oxidation found for human hemoglobin prior to saturation of the "high-affinity" site (4) and has recently been investigated in greater detail by Louro and Tabak [17].

These effects can now be understood in terms of the room temperature cross-linked 
"high-affinity" site [15] which places the Cu(II) near the sulfhydryl and in the region of the "low-affinity" $\mathrm{Cu}$ (II) binding site.

In fact, it has been suggested [15] that $\beta$-143 histidine, which has been implicated in binding to the "low-affinity" site [3], is also part of this "high-affinity" site. The location of the $\beta-143$ histidine on the surface of the molecule suggests that while it may be part of the "low-affinity" site directly involved in the oxidation (site 2), it is perhaps crucial for the initial site on the surface of the protein (site 1), which is a "low-affinity" site that is available when the sulfhydryl groups are blocked.

This hypothesis is consistent with the effect of NEM on the binding of Cu(II) to the "low-affinity"' site (Fig. 7) in human hemoglobin. NEM, which produces only a $20 \%$ decrease in the equilibrium constant for the "low-affinity" site of horse hemoglobin, produces a much more dramatic decrease in the binding of $\mathrm{Cu}$ (II) to the low-affinity site of human hemoglobin. Furthermore, the failure to detect an additional decrease in signal intensity for human hemoglobin at $\mathrm{Cu}$ /heme ratios greater than 0.5 suggests that for human hemoglobin with the sulfhydryl blocked by a spin label both "low-affinity" sites found with horse hemoglobin (sites 1 and 2 ) are essentially nonexistent.

The effect of $\mathrm{Cu}$ (II) binding to the "high-affinity" site on the "low-affinity" site can thus be explained in terms of a disruption of the equilibrium between site 1 on the surface and site 2 in the pocket, so that only site 2 can be populated. The spin label studies and the dipolar effect between $\mathrm{Cu}$ (II) and $\mathrm{Fe}$ (III) further suggest that in human hemoglobin, perhaps because of the $\mathrm{Cu}$ (II) bound to site 4 , some further perturbation occurs in the region of site 2 so that the copper-iron distance is further reduced.

\section{CONCLUSION}

A summary of the description of the cupric ion binding sites are as follows:

Site 1: 9-13 $\AA$ from nitroxide reacted $\beta-93$ cysteine; nitrogen and oxygen ligands on surface near cys $\beta-93$; rearranges to form site 2 prior to oxidation; observed at all temperatures; weak binding.

Site 2 : $<10 \AA$ from heme; requires free $\beta$-93cys, may involve coordination to cysteine; involved in the oxidation of $\beta$-heme; observed at all temperatures; weak binding.

Site 3: $\sim 17 \AA$ from nitroxide reacted Cys $\beta-93$; four nitrogen donor atoms near the $\mathrm{N}$-terminal including $\beta-2$ histidine and $\beta-1$ valine; not involved in the oxidation of $\beta$ heme; observed at low temperatures $\sim 77 \mathrm{~K}$; strong binding.

Site 4: $\sim 7 \AA$ from nitroxide reacted Cys $\beta-93$; four nitrogen donor atoms due to cross-linking of two $\beta$-subunits; blocks site 1 ; not involved in the oxidation of $\beta$-heme; observed at $298 \mathrm{~K}$; strong binding.

The close proximity of sites 2 and 4 , which should both be populated upon the addition of four equivalents of cupric ion per hemoglobin molecule, suggests that a study of these sites may be pertinent to understand the origin of "type 3" cupric complexes, which consist of a pair of cupric ions in close proximity and strongly antiferromagnetically coupled [18]. It is also intriguing to try to involve the cysteine residue $93 \beta$ in the binding to site 1 since it is located close to the metal centers and its free -SH group is required for oxidation of the $\beta$ heme by copper [3]. Similar relationships between heme iron, -SH group, and copper are found in cytochrome oxidase. Thus, the $\beta$ subunit within the hemoglobin molecule may be a general model to study the interactions between two or three metal centers and - $\mathrm{SH}$ groups for the redox activity of proteins. 
The authors express their appreciation to Dr. G. L. Eichhorn and Dr. J. S. Hyde for their advice and encouragement and to Ms. Jane Heim for technical assistance. Part of this work at the National Biomedical ESR Center was supported by NIH grants AM-15770 and RR-01008.

\section{REFERENCES}

1. F. Taketa and W. E. Antholine, J. Inorg. Biochem. 17, 109 (1982).

2. W. E. Antholine, R. Basosi, J. S. Hyde, and F. Taketa, J. Inorg. Biochem. 21, 69 (1984).

3. J. M. Rifkind, Copper and the Oxidation of Hemoglobins, in Metal Ions in Biological Systems, Vol. 12, Helmut Sigel, Ed., Dekker, New York, 1981.

4. J. M. Rifkind, L. D. Lauer, S. C. Chiang, and N. C. Li, Biochemistry 15(24), 5337 (1976).

5. P. T. Manoharan, K. Alston, and J. M. Rifkind, Bull. Mag. Res. 5, 255 (1983).

6. G. Bemski, T. Arends, and G. Blanc, Biochem. Biophys. Res. Commun. 35, 599 (1969).

7. J. S. Leigh, Jr., J. Chem. Phys. 52(5), 2608 (1970).

8. J. P. Jesson, The Paramagnetic Shift, in NMR of Paramagnetic Molecules: Principles and Applications, A. M. La Mar, W. Dew Horrocks, Jr., and R. H. Holm. Eds., Academic, New York, 1973, p. 38.

9. J. M. Rifkind, Biochemistry 18(18), 3860 (1979).

10. K. S. Iyer, S.-J. Lau, S. H. Laurie, and B. Sarkar, Biochem. J. 169, 71 (1978).

11. J. M. Rifkind and J. Heim, unpublished results.

12. M. Perutz and A. Fermi, Protein Data Bank, University Chemical Laboratory, Lensfield, Cambridge, England or Brookhaven National Laboratory, Upton, New York.

13. R. Basosi, W. E. Antholine, W. Froncisz, and J. S. Hyde, manuscript submitted.

14. W. E. Antholine, G. Riedy, J. S. Hyde, R. Basosi, and D. H. Petering, J. Biomole. Struct. Dynamics, in press.

15. M. Tabak and S. R. W. Louro, private communication.

16. R. F. Campbell and J. H. Freed, J. Phys. Chem. 84, 2668 (1980).

17. S. R. W. Louro and M. Tabak, private communication.

18. J. R. Boas, J. R. Pilbrow, and T. D. Smith, ESR of Copper in Biological Systems, in Biological Magnetic Resonance, Vol. 1, L. J. Berliner and J. Reuben, Eds., Plenum, New York, 1978.

Received March 29, 1985; accepted May 13, 1985 